\title{
10 Jahre G-DRGs - ein kurzer Rückblick
}

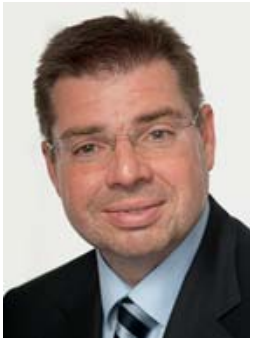

D. C. Wirtz

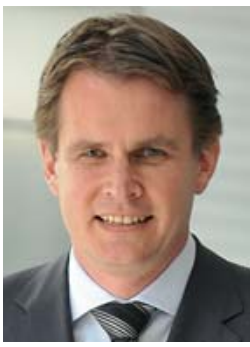

U. Stöckle

Bibliografie

DOI http://dx.doi.org/

10.1055/s-0034-1368570

Z Orthop Unfall 2014; 152: 222

223 @ Georg Thieme Verlag KG Stuttgart · New York .

ISSN 1864-6697

Korrespondenzadressen Univ.-Prof. Dr. med.

Dieter C. Wirtz

Direktor der Klinik und Poliklinik für Orthopädie und Unfallchirurgie Universitätsklinikum Bonn Sigmund-Freud-Straße 25 53105 Bonn

Tel.: 0228/287-14170 Fax: 0228/287-14175

dieter.wirtz@ukb.uni-bonn.de

Univ.-Prof. Dr. Ulrich Stöckle Ärztlicher Direktor BGU Klinik Tübingen Schnarrenbergstraße 96 72076 Tübingen

Tel.: 07071/606-1001

Fax: 07071/606-1002

ustoeckle@bgu-tuebingen.de

\section{Liebe Leserinnen und Leser \\ $\nabla$}

immer mehr Publikationsmanuskripte erreichen uns mit gesundheitsökonomischen Fragestellungen. So werden auch in diesem Heft 2 Arbeiten zu den Themen „Kosten-Nutzen-Analyse der Schnittbildgebung beim Schädelhirntrauma“ und „Kosten-Deckungsanalyse der Ballonkyphoplastie“" publiziert. Aus Editorensicht ist diese Entwicklung sehr zu begrüßen, weil es zeigt, dass Orthopäden und Unfallchirurgen sich auch wissenschaftlich mit Fragen zur Wirtschaftlichkeit ihres Handelns immer mehr befassen. Zehn Jahre nach Einführung der G-DRGs muss jedem bewusst sein, dass wenn wir uns nicht nur von Ökonomen und Unternehmensberatern leiten und lenken lassen wollen, dann brauchen wir klare Aussagen und entsprechende Kenntnisse darüber, welche Diagnostik und welche Therapie medizinisch notwendig, aber auch bezahlbar ist.

Blickt man nun auf die vergangenen 10 Jahre DRG-System zurück, so zeigen sich unterschiedliche Effekte, die sowohl als positiv aber auch als negativ zu werten sind:

Das DRG-System mit dem Grundprinzip „gleiches Geld für gleiche Leistung“" hat zur intensiven und auch vermehrt transparenten Auseinandersetzung mit Abläufen, Prozessen und Kosten geführt. Die systemimmanenten Anreize haben in den meisten Kliniken zur Steigerung der Fallzahlen und gleichzeitig zur Reduktion der Verweildauer beigetragen. Ob dadurch aber auch die Effizienz gebessert wurde, bleibt fraglich. Nach einer KPMG-Studie, bei der die Ergebnisqualität der Gesundheitssysteme europäischer Staaten in Relation zum Anteil der Gesundheitsausgaben am jeweiligen Bruttosozialprodukt berechnet wurde, rangiert Deutschland gerade mal auf Platz $17 \mathrm{im}$ unteren Mittelfeld [1]. Dieser „niedrige Effizienzindex" beruht dabei nicht so sehr auf einer qualitativ „nicht hochwertigen“ Behandlung, sondern auf den im Vergleich zu den anderen europäischen Ländern extrem hohen Ausgaben (in 2011 rund 294 Milliarden Euro) [1].

Ein Grund für diese Ausgabensituation ist die Steigerung im Personalkostenbereich. Bedingt durch die Leistungsverdichtung ist die Anzahl der Klinikärzte (2003: 114105 Vollzeitstellen; 2011: 138955 Vollzeitstellen) deutlich gestiegen. Darüber hinaus ist aber auch die Zahl der Mitarbeiter im Verwaltungsdienst (2003: 57927 Vollzeitstellen; 2011: 59759 Vollzeitstellen) gestiegen [2]. Neue Berufsgruppen wie Kodierfachkräfte, Betten-, Patienten- und Casemanager ha- ben ihren Einzug in die Kliniken gefunden. Leistungsdokumentation, Kodierung, Abrechungsvorbereitung und Plausibilisierung der Daten sind immer aufwendiger geworden. Was hingegen reduziert wurde, sind Pflegestellen: lag die Zahl der Pflegekräfte in 2003 bei 320158 Vollzeitstellen, so lag diese in 2011 nur noch bei 310817 Vollzeitstellen [2]. Dies bedeutet bei gegebener Fallzahlsteigerung letztendlich eine deutliche Reduktion der pflegeseitigen Zuwendung zum Patienten, was jedoch gerade bei dem zunehmend alternden und multimorbiden Patientenkollektiv nicht Zielsetzung eines sozialen Gesundheitssystems sein sollte.

Ein weiterhin zunehmendes Problem in unserem Gesundheitssystem ist in der universitären Medizin zu sehen. Schon nach einigen Jahren „Laufzeit" des DRG-Systems zeigte sich gerade für die Spitzenmedizin ein nicht tragfähiges Auseinandergehen der Schere zwischen Erlösen und Kosten [3]. Bedingt ist dies vornehmlich durch die Häufung von Extremkostenfällen, die Vorhaltung einer alle Disziplinen umfassenden Notfallversorgung in ständiger Bereitschaft und die Erstanwendung neuer medizinischer Produkte und Verfahren. Dazu kommt die genuine universitäre Aufgaben in Forschung und Lehre, die zwar durch die Zuführungsbeträge der Länder finanziert wird, in der gegenwärtigen Praxis werden diese jedoch häufig zur Deckung der klinischen Budgetdefizite herangezogen. Die Medizinforschung an deutschen Standorten wird auf diese Weise gegenüber vergleichbaren Institutionen im Ausland eindeutig benachteiligt [4]. Dass es dabei auch innerhalb der Universitätsklinika zu zunehmenden Spannungen zwischen „Klinikum“ und „Fakultät“ kommt, ist leider an vielen Standorten spürbar. Nach Angaben des Jahresberichts des Verbands der Universitätsklinika Deutschlands (VUD) sind etwa die Hälfte der 33 Unikliniken existenziell bedroht. Insgesamt betrage das Jahresdefizit im vergangenen Jahr (2013) 161 Millionen Euro [4].

Kommen dann künftig noch Tarifänderungen, höhere Kosten für Energie oder Versicherungsleistungen hinzu, so wird klar, dass ein DRG-System, welches in Bezug auf diese Aspekte über Jahre hinweg nicht oder nur verzögert „lernt“, einer dringenden Überarbeitung bzw. Abänderung bedarf. So wie die Bundesärztekammer aktuell gefordert hat [5], sollten die DRGs als Basis der künftigen Krankenhausvergütung ergänzt werden durch die Möglichkeit einer individuellen leistungsadaptierten Budgetfestlegung. Dabei ist das politisch gewollte Prinzip „pay-for-performance“ 
sicherlich als richtiger Ansatz zu werten, allerdings muss eine patientenbezogene Risikoadjustierung mit Berücksichtigung von Komorbiditäten und Compliance erfolgen. Insbesondere für Patienten, die hohe Kosten verursachen, muss ein tragfähiges Konzept für eine aufwandsgerechte Vergütung etabliert werden. Spitzenmedizin in einem „industrialisierten Umfeld“, wo nur das durchschnittliche Preis-Mengen-Gerüst und der Billig-Einheitstarif zählt, ist nicht machbar.

D.C. Wirtz, U. Stöckle

\section{Literatur}

1 Kaiser T. Das deutsche Gesundheitssystem ist das Geld nicht wert. Die Welt 20.01.2014. Im Internet: http://www.welt.de/wirtschaft/article 124010016

2 Osterloh F. 10 Jahre DRG-System. Mehr Ärzte, mehr Kodierkräfte. Deutsches Ärzteblatt 2014; 10: B330

3 Peters J. Vom Untergang der Unikliniken. Frankurter Allgemeine Zeitung, Wissenschaft, 04.11.2009

4 Richter-Kuhlmann E. Sonderweg Universitätsklinika. Deutsches Ärzteblatt 2014; Heft 11: B373-374

5 Flintrop J, Gerst T. Qualität im Fokus. Deutsches Ärzteblatt 2014; 7: B211-B213 